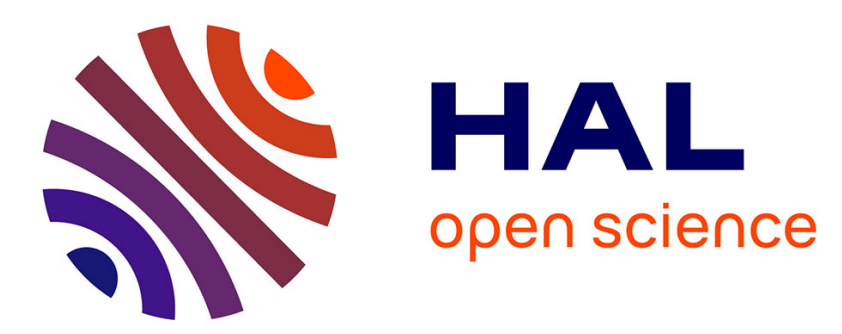

\title{
Forces and flow induced by a moving intruder in a granular packing: coarse-graining and DEM simulations versus experiments
}

Julien Lehuen, Jean-Yves Delenne, Agnès Duri, Thierry Ruiz

\section{To cite this version:}

Julien Lehuen, Jean-Yves Delenne, Agnès Duri, Thierry Ruiz. Forces and flow induced by a moving intruder in a granular packing: coarse-graining and DEM simulations versus experiments. Granular Matter, 2020, 22 (4), 10.1007/s10035-020- . hal-02936331

\section{HAL Id: hal-02936331 \\ https://hal.inrae.fr/hal-02936331}

Submitted on 11 Sep 2020

HAL is a multi-disciplinary open access archive for the deposit and dissemination of scientific research documents, whether they are published or not. The documents may come from teaching and research institutions in France or abroad, or from public or private research centers.
L'archive ouverte pluridisciplinaire $\mathbf{H A L}$, est destinée au dépôt et à la diffusion de documents scientifiques de niveau recherche, publiés ou non, émanant des établissements d'enseignement et de recherche français ou étrangers, des laboratoires publics ou privés. 


\title{
Forces and flow induced by a moving intruder in a granular packing: coarse-graining and DEM simulations versus experiments
}

\author{
Julien Lehuen ${ }^{1,2^{*}}$, Jean-Yves Delenne ${ }^{2}$, Agnès Duri ${ }^{2}$, Thierry Ruiz ${ }^{1}$ \\ ${ }^{1}$ QualiSud, Univ Montpellier, CIRAD, Montpellier SupAgro, Univ Avignon, Univ La Réunion, Montpellier, \\ France. \\ ${ }^{2}$ IATE, Univ Montpellier, CIRAD, INRA, Montpellier SupAgro, Montpellier, France. \\ * correspondant author
}

Published in Granular Matter 22, 78 (2020). https://doi.org/10.1007/s10035-02001047-5

\begin{abstract}
.
The study of stress transmission and particles' mobility in powder beds constitutes a challenging issue to improve processes such as milling, agglomeration and kneading. In this paper the ability to promote particle mobilities by means of an intruder is investigated. An experimental setup was developed to characterize the particles' mobility at the voxel scale. A Particle Image Velocimetry (PIV) algorithm was employed to determine the velocity fields in the vicinity of an intruder in ascendant vertical motion. A Discrete Element Method (DEM) model was developed to simulate the same system at the particle scale. Velocity maps were determined relying on a coarse-graining procedure and compared to PIV results. During the rise of the intruder the experimental and simulated drag forces are in good agreement. Spatiotemporal correlation between the granular mobility and the state of the force network are analyzed as a function of the height of the intruder. The drag force profile exhibits a relaxation
\end{abstract}


trend, noised by fluctuations whose origin are in successive loading/rupture events. An interpretation of these fluctuations is proposed with regard to the development of preferential paths in the normal force network, and to the localization of sliding at the contacts scale. Finally, we show that global strains are a consequence of the ejection of particles directly in contact with the ends of the intruder.

Keywords. Intruder, Granular bed, Drag force, Particle Image Velocimetry (PIV), Discrete element method (DEM), Coarse graining.

\section{Introduction}

Mixing powders using intruders, such as blades, paddles, agitators... is widely used in chemical engineering [1,2], civil engineering [3], pharmaceutical [4,5], food science [6,7] or agronomy [8,9]. Common concerns regarding interactions between an intruder and a granular bed are shared by many unit operations like blending, kneading [10], wet agglomeration $[11,12]$ or milling $[13,14]$.

More precisely, in blending operations, the paddle transmits mechanical energy to the granular bed inside a vessel [15]. To control the input energy or the shear rate, a range of low to high blenders are available. The efficiency of shear transmission requires adapted design of the whole setup (paddle shape and trajectory, vessel, wall-paddle gap) [16,17]. One major issue is to transfer energy from the scale of the reactor to the scale of the micro-structure. At the particle scale, this energy is dissipated through frictional contacts and damping. In addition of these interactions the available energy is turn into particle motion. Compared to liquids, the control 
of particle flows is a challenging issue due to spatial (localization of the flow, shear banding) and temporal (intermittent flow) deviations of particles from the mean field. In the case of mixing, the blender is used to promote the mobility of particles compared to their mutual friction whereas friction is rather privileged in agglomeration or kneading operations to control the growth in size of aggregates $[18,19]$. Understanding how energy is distributed at the particle scale to promote local friction or mobility is a key issue to optimize the processes.

In these reactors shearing is promoted by a blade or paddle that is cyclically driven into and out of the granular bed [16-17]. Most of the mechanical energy inducing particles mobility is transferred during pull-off phases. Penetration phase can be analyzed based on studies performed in other scientific fields regarding intruder penetration in a granular packing [2025]. However, to our best knowledge, the pull-off phase has been little studied per se in spite of its interest in process engineering. In this framework we will consider a simplified configuration in which the intruder is removed vertically at constant speed from the granular bed.

In this paper we propose a methodology to investigate the influence of an intruder on force transmission and particle mobility in an ensiled granular bed. This methodology associates experiments using an original setup and 2D Discrete Element Methods (DEM) simulations. In the experiments we use a transparent glass cell filled with glass beads. The pull-off test consists in moving an intruder upward at constant speed into the granular bed. The drag force acting on the intruder is measured while a slow-motion camera records the displacements of the particles around the intruder. Relying on a Particle Image Velocimetry (PIV) method it is possible to determine the $2 \mathrm{D}$ experimental velocity fields for particles behind the front wall. Numerical simulations were performed at same velocity on a similar configuration of cell, packing and 
intruder. One major interest in simulation is that it gives detailed information on force transmission that is hardly accessible by experiments. Particular attention was paid to the filling procedure which is important to control the initial microstructure of the packing. During the simulations, we monitored the particle velocities, and the normal and tangential forces as a function of the height of the intruder. This data is then used in a coarse-graining approach to upscale the velocity field. One of the main objectives was to provide comparable experimental and numerical systems in which the walls have an influence on the local texture of the packing as in mixing or blending problems. In this regard, experimental and numerical drag forces and velocity fields are compared in this paper. There is a large corpus of references on granular materials, which deals with experimental, theoretical and numerical aspects. One major achievement was to clarify the mechanical behavior of granular flow with the $\mu(\mathrm{I})$ rheology [26]. However, many transient phenomena remain poorly investigated despite their great interest in technical applications for example in chemical and civil engineering. One critical issue is to consider the effect of non-homogeneous boundary conditions. While many papers deal with the penetration of an intruder in a packing $[23,24,44]$, to our best knowledge there are very few that deal with its pull-off [27]. Note that these are far to be symmetrical configurations which confirm the difference of behavior with our configuration and show the need for a specific study. In our case an intermittent flow around the intruder was evidenced generating strong fluctuations in the drag force profile during the motion of the intruder. These fluctuations were already investigated in previous studies on intruder penetration in a granular medium [28] and have been attributed to dilatancy phenomenon. We discuss the relevance of this interpretation for our experiment and finally, we describe the evolution of the flowing zones in relation to force fluctuations and sliding contacts. 


\section{Materials and methods}

This section, describes the experimental and numerical setups developed in order to study the behavior of a packing during the vertical ascension of an intruder.

\subsection{Experimental device}

The device consists of an open rectangular cell of length $L_{c}=100 \mathrm{~mm}$, width $W_{c}=50 \mathrm{~mm}$ and height $H_{c}=500 \mathrm{~mm}$ made with transparent glass walls (Fig. 1). A rigid rectangular intruder of length $L=10 \mathrm{~mm}$, width $W=49 \mathrm{~mm}$ is preliminary positioned at the bottom of the cell and used to probe the flow properties of the particle bed. Before the test, the cell was filled up to a height of $150 \mathrm{~mm}$ with glass beads of size $\bar{d}=3 \pm 0.05 \mathrm{~mm}$, with a density $\rho=$ $2500 \mathrm{~kg} \mathrm{~m}^{-3}$ and bulk modulus $E=64 \mathrm{GPa}$. Following Ovarlez et al. [29], the coefficients of friction between the grains and a glass surface $(\mu)$ was determined using three grains glued on a metal plate forming a tripod. This tripod was disposed on a glass plate (same material as the cell wall) equipped with an inclinometer. The glass plate was gradually tilted up to a sliding friction angle $\theta\left(26.5^{\circ}\right)$ at which the tripod slips. Even if the obtained value may largely depends on surface roughness [30], we chose to use the same coefficient of friction for the contacts between the particles $\mu=0.5 \pm 0.02$.

To control the filling rate a hopper of aperture diameter $D_{p}=11 \mathrm{~mm}$ is used with an angle chosen sufficiently large to be greater than the repose angle of the granular bed. The outlet of the hopper is positioned at a height $H_{h}=447 \mathrm{~mm}$. During the test, the intruder is pulled off from the granular material at a constant velocity $V$. Although in the experiments, the velocity may be varied in the range $\left[1-29 \mathrm{~mm} \mathrm{~s}^{-1}\right]$, in this study, we focus on the results obtained for $V=1.2 \mathrm{~mm} \mathrm{~s}^{-1}$. The drag force is measured using a force sensor (TME-Type F $108 \mathrm{TC}$-range: 
1-25 daN) attached to the intruder via a steel rod. We checked that the force on this shaft is below $1.6 \%$ of the drag-force in the worst-case (at the beginning of the test). To capture the particle motion in the vicinity of the intruder we use a high-speed camera (Dalsa CMOS monochrome, frequency rate $100 \mathrm{fps}$, image resolution 1392 x 1024 pixels) whose displacement follows that of the intruder. A Particle Image Velocimetry (PIV) method (described in the next section) is employed to determine as a function of time, the $2 \mathrm{D}$ velocity field at the surface of the front wall (Fig. 1c).

a)

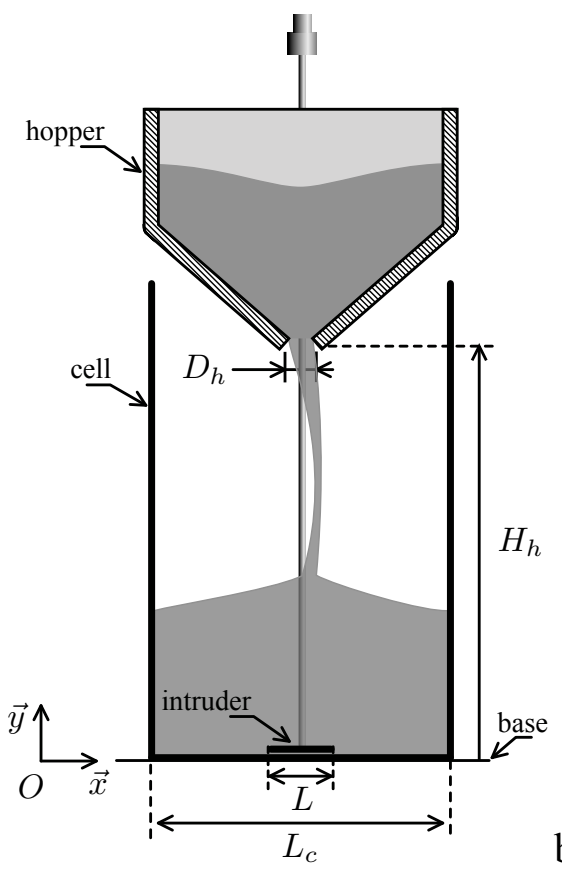

b)

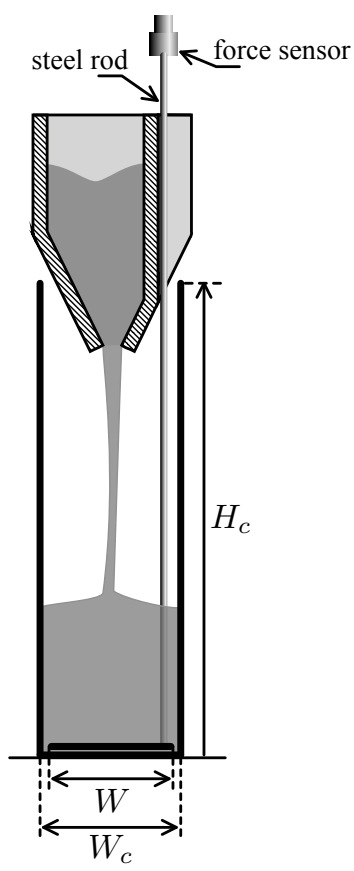

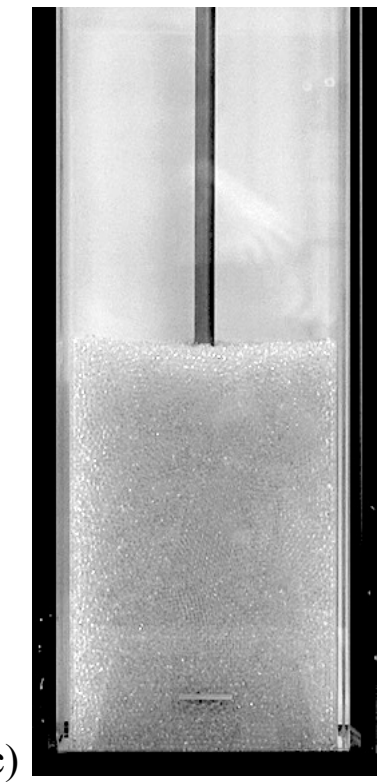

Fig. 1 Experimental device: a) front face; b) lateral face; c) experimental cell (for better visualization, the photograph is taken with an intruder positioned at $20 \mathrm{~mm}$ from the base).

\subsection{Particle Image Velocimetry}

The 2D velocity field is determined thanks to the PIVlab ${ }^{\circledR}$ module of Matlab ${ }^{\circledR}$ which provides useful tools for Particle Image Velocimetry (PIV). In our study we employed a Fast Fourier Transformation (FFT) correlation algorithm with multiple passes and deforming windows of 64x64 and 32x32 pixels [31]. The images were captured using the camera at a frame rate 
$\Delta t_{P I V}=50 \mathrm{~ms}$. In order to follow the particle flow in the frame of the intruder, the trajectory of the camera was set to be the same as the intruder and a fixed mask zone was defined around its boundary. To exclude the walls and the free surface in the analysis, we defined a Region Of Interest (ROI) localized on the granular sample. With this setup an individual glass bead is described approximately with 35 pixels which corresponds to a spatial resolution of $1.3 \bar{d}$. The velocity field $\vec{v}(x, y)=v_{x} \vec{x}+v_{y} \vec{y}$ is then determined as a function of time during the intruder pull-off. In the following we only focus on maps of velocity magnitude $\left(\sqrt{v_{x}^{2}+v_{y}^{2}}\right)$.

\subsection{Numerical method}

In order to better understand the mechanisms involved during experiments, we developed a versatile $2 \mathrm{D} \mathrm{C}++$ code based on the framework of the Discrete Element Method (DEM) [32]. We used a classical molecular-dynamics approach with an explicit velocity Verlet integration scheme [33]. The main characteristics of the code are briefly recalled in this section.

\section{Contact forces between particles}

In the simulations the particles are 2D disks interacting via frictional elastic contact laws. Fig. 2a shows a particle $i$ of center of mass $O_{i}$ and diameter $d_{i}$, located at a position $\vec{r}_{i}$ and with a velocity $\vec{v}_{i}$ and an angular velocity $\dot{\theta}_{i}$. Figure $2 \mathrm{~b}$ shows the contact between two particles $i$ and $j$ of center of mass $O_{i}$ and $O_{j}$ located at $\vec{r}_{i}$ and $\vec{r}_{j}$ (in the following for sake of simplicity we avoid to mention $i$ and $j$ in pairwise variables). The distance between particles is determined using $\delta_{n}=\left\|\vec{r}_{i}-\vec{r}_{j}\right\|-\frac{1}{2}\left(d_{i}+d_{j}\right)$. For particles in contact $\left(\delta_{n} \leq 0\right)$ we define $\vec{n}=\frac{\vec{r}_{i}-\vec{r}_{j}}{\left\|\vec{r}_{i}-\vec{r}_{j}\right\|}$ the normal vector at contact and $\vec{t}$ the tangential vector chosen so that $(\vec{n}, \vec{t})$ is right-handed. In this 
local frame of reference, the contact force can be written as $\vec{F}=F_{n} \vec{n}+F_{t} \vec{t}$, where $F_{n}$ and $F_{t}$ are the normal and tangential components, respectively.

a)

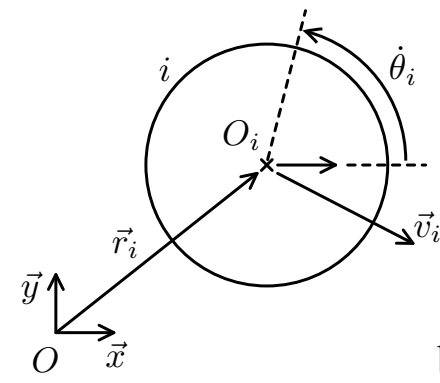

b)

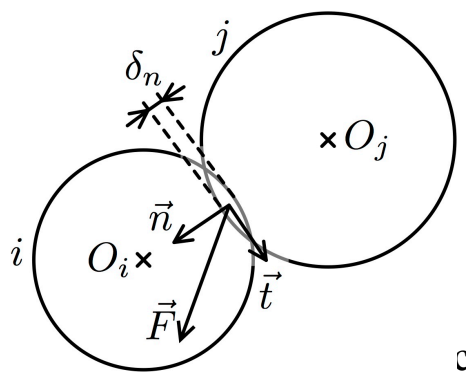

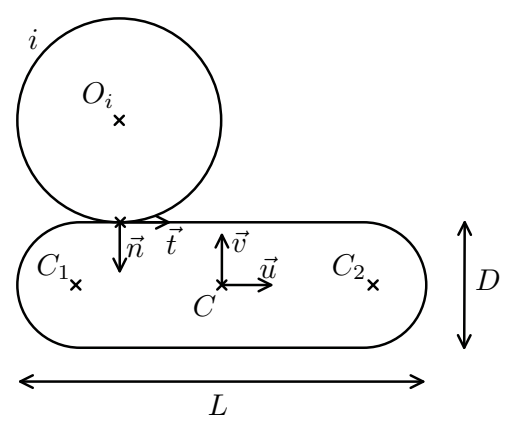

Fig. 2 a) Kinematic coordinates of a particle $i$ in the global frame of reference; b)

Contact between two particles $\mathrm{i}$ and $\mathrm{j}$ as revealed by an overlap equals to $\left.\delta \_n ; \mathrm{c}\right)$

Contact between a particle and an intrudeur.

$F_{n}$ is determined using a visco-elastic law composed of two additive terms $F_{n}=F_{n}^{e}+F_{n}^{v}$, where $F_{n}^{e}$ and $F_{n}^{v}$ are the elastic and viscous contribution, respectively. For the elastic part we used a linear (unilateral) elastic law $F_{n}^{e}=-k_{n} \delta_{n}$, where $k_{n}$ is the contact stiffness. Following Rognon et al. [34] we set $k_{n} \sim E \bar{d}$ (corresponding to Hertzian contact in 2D), with $E$ the elastic modulus and $\bar{d}$ is the average diameter of all particles. For the viscous part, used to dissipate energy during collisions, we chose $F_{n}^{v}=v v_{n}$, where $v$ is the damping coefficient and $v_{n}=\left(\vec{v}_{i}-\vec{v}_{j}\right)$. $\vec{n}$ is the relative normal velocity between the two particles. In the absence of cohesion, this damping coefficient is related to the normal coefficient of restitution $e$ through the equation $v=1 / \sqrt{1+(\pi / \ln e)^{2}}[35]$.

For the friction force $F_{t}$, we used a viscous-regularized Coulomb law [36]: $\left|F_{t}\right| \leq F_{t}^{\text {slip }}$ with $F_{t}^{\text {slip }}=\mu F_{n}$ and $\mu$ the friction coefficient. For each time step $\Delta t$, an increment of tangential force opposed to the increment of the tangential relative displacement $\delta_{t}$ is computed such as $\delta F_{t}^{\Delta \mathrm{t}}=-k_{t} \delta_{t}$ with $k_{t}$ the tangential stiffness of the contact. At time $t+\Delta t$, the tangent force is equal to $F_{t}^{\mathrm{t}+\Delta \mathrm{t}}=F_{t}^{t}+\delta F_{t}^{\Delta \mathrm{t}}$ [37] where $F_{t}$ is limited by the Coulomb law (Fig. 3). 


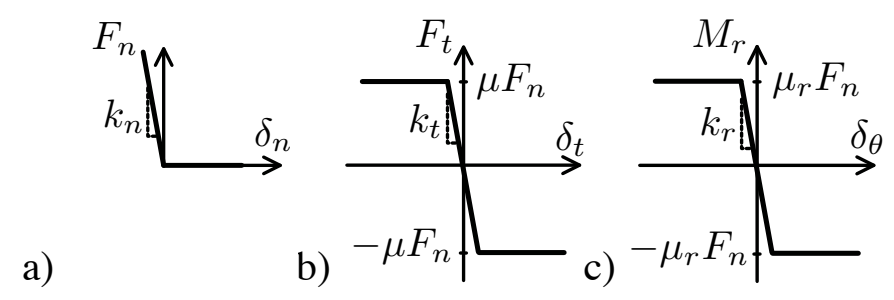

Fig. 3 Components of the regularized friction law a) contact, b) sliding, c) rolling. According to Campbell [38], $k_{t}$ has a weak influence for cohesionless particle and is fixed to $\frac{k_{n}}{2}$ in this paper.

For the rolling moment $M_{r}$, we used a rolling friction law (Fig. 3c): $\left|\mathrm{M}_{\mathrm{r}}\right| \leq \mu_{r} F_{n}$ with $\mu_{r}=$ $\mu \bar{d} 10^{-2}$ the rolling friction coefficient. For each time step $\Delta t$, an increment of moment opposed to the increment of the rotational relative displacement $\delta_{\theta}$ is computed such as $\delta \mathrm{M}_{r}^{\Delta t}=-k_{r} \delta \theta$ with $k_{r}=\bar{d}^{2} k_{n} 10^{-4}$ the rotationnal stiffness chosen following the paper of Gilabert et al. [35]. At time $t+\Delta t$, the rolling moment is equal to $\mathrm{M}_{r}^{t+\Delta t}=\mathrm{M}_{r}^{t}+\delta \mathrm{M}_{r}^{\Delta t} \quad$ where $\mathrm{M}_{\mathrm{r}}$ is limited by the rolling friction law. Apart from $k_{t}, k_{r}$ and $\mu_{r}$, all other parameters are chosen according to experimental data described in section 2.1.

\section{Walls and intruder}

Following the article of Fakih et al [9], for the boundary conditions (wall and intruder) we use sphero-line objects that are constituted of a line connecting two spherical caps. The contact forces between particles and these objects are obtained using similar equations as described for particle's contact. As an example, Fig. 2c shows the contact between the intruder and a particle. Different cases can be considered depending on the position of this contact. For that we define a system coordinate $(\vec{u}, \vec{v})$ attached to the sphero-line, where $\vec{u}=\overrightarrow{C_{1} C_{2}} /\left\|\overrightarrow{C_{1} C_{2}}\right\|$ is an unitary vector orienting the sphero-line object and $\vec{v}$ is the right-handed oriented unitary vector. The 
overlap can be calculated as a function of the position of the contact based on the following equations:

- If $\overrightarrow{C O_{\mathrm{i}}} \cdot \vec{u} \leq-C_{1} C_{2} / 2$ then $\delta_{n}=\left\|\overrightarrow{O_{\mathrm{i}} C_{1}}\right\|-\frac{\mathrm{d}_{\mathrm{i}}+\mathrm{D}}{2}$

- If $\overrightarrow{C O_{\mathrm{i}}} \cdot \vec{u} \geq C_{1} C_{2} / 2$ then $\delta_{n}=\left\|\overrightarrow{O_{\mathrm{i}} C_{2}}\right\|-\frac{\mathrm{d}_{\mathrm{i}}+\mathrm{D}}{2}$

- If $-C_{1} C_{2} / 2 \leq \overrightarrow{C 0_{\mathrm{i}}} \cdot \vec{u} \leq C_{1} C_{2} / 2$ then $\delta_{n}=\left|\overrightarrow{C 0_{\mathrm{i}}} \cdot \vec{v}\right|-\frac{\mathrm{d}_{\mathrm{i}}+\mathrm{D}}{2}$

For the computation of the contact between particles and the intruder, we use the same relationships as described above for particle-particle contacts.

\subsection{Numerical tests}

\section{Sample preparation}

The numerical cell as the same dimensions as the experimental one, except that the numerical simulations are performed in $2 \mathrm{D}$. For the simulations we used a slight polydispersity $\left(\frac{d_{\max }}{d_{\min }}=\right.$ 2) in order to limit crystallization effects while remaining as closed as possible to experimental value of $\bar{d}$ [39]. For the particle-particle and particle-wall normal coefficients of restitution we set $e=0.9$ [40] which corresponds to a damping $v=0.034 \mathrm{Nsm}^{-1}$. All simulations are done with a timestep $\Delta t=10^{-7} \mathrm{~s}$. Assuming $\Delta t_{c} \approx \sqrt{\frac{m_{\min }}{k_{n}}}$ (where $m_{\min }$ is the mass of the lightest particle and $k_{n}=E \bar{d}=1.9210^{8} \mathrm{Nm}^{-1}$ ) we have $\Delta t / \Delta t_{c} \approx 1 / 60$. 
The sample preparation consists in two steps. The particles are first placed on a triangular mesh and settled into a hopper under gravity (Fig. 4a). To obtain the same height $H_{b}=150 \mathrm{~mm}$ of granular bed as in experiment we used 1600 particles. The simulation runs until relaxation of the packing ( $1 \mathrm{~h}$ on an Intel Core i7 CPU). An hopper angle of $\sim 50^{\circ}$ is chosen to promote particle flowing. We then let the particles flow through the aperture whose size allows to control the filling rate (Fig. $4 \mathrm{~b}$ ). It takes about $3 \mathrm{~h}$ to fill the cell and reach a full stabilization of the bed. At the end of this process the particle solid fraction $\Phi \approx 0.82 \pm 0.02$.

\section{Pull-off test}

The numerical test consists in vertically pulling the intruder off the granular bed (Fig. 4c) at constant velocity $V$. Although, simulations were performed using different intruder velocities, in this study we compared with the experiments for a single value of $V=1.2 \mathrm{mms}^{-1}$, corresponding to approximatively $0.7 \%$ of the characteristic velocity $\sqrt{g \bar{d}}$ where $g=9.81 \mathrm{~ms}^{-}$

${ }^{2}$. As this value is very low we expect that the flow regime will not directly depends on the magnitude of the velocity of the intruder. In other words, this case can be viewed as a limit case in which the role of the intruder is to structure the packing without inertial contribution. By way of example the lowest velocities employed in low-shear mixing are about ten times higher [41]. Note however that due to rupture of force chains the particles experience local intermittent flows and cannot be considered as in quasi-static regime. During the full simulation which last more than $300 \mathrm{~h}$ of computation time, we periodically record the average force on the intruder (called drag force in the following), the positions and velocities of particles, and the normal and tangential force networks. 


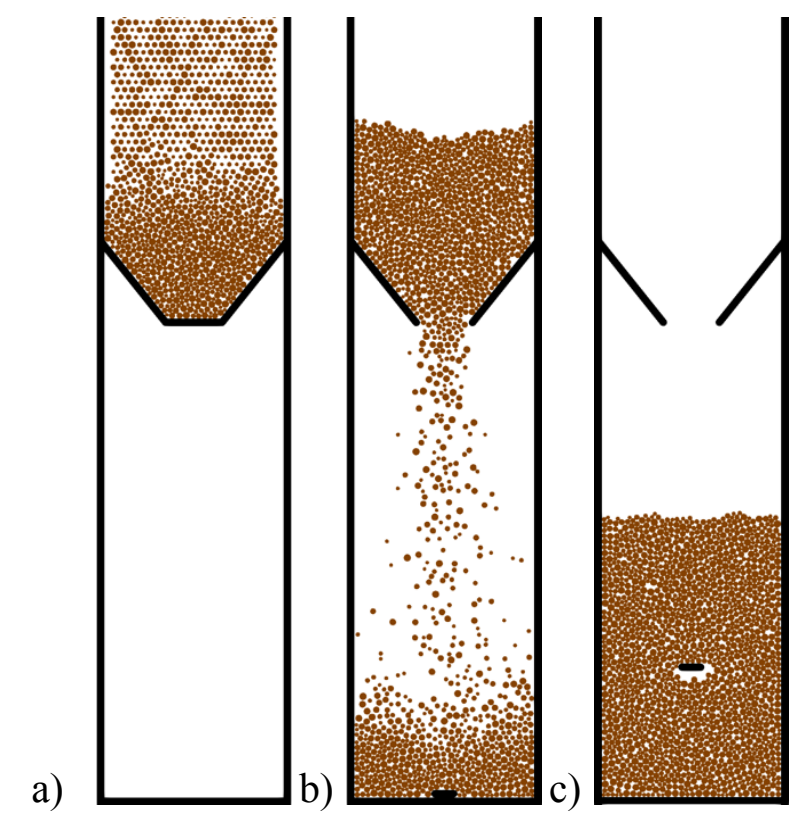

Fig. 4 Numerical device: a) particles are initially placed on a triangular lattice mesh and settled in the hopper; b) filling of the cell; c) pull-off of the intruder.

\section{Coarse graining}

Following the works of Weinhart et al [42] and Algarra et al. [43] we employed a coarse graining method to upscale the continuous velocity field from the positions of the particles and their velocities computed by DEM. Among other, this method has the advantage to satisfy the conservation equations of continuum mechanic. Fig. 5a shows the DEM domain meshed using a rectilinear grid of spatial resolution $\Delta x$. In the following we chose $\Delta x=\frac{\bar{d}}{8}$ which gives a good compromise between the resolution of the mesh and the computation time (250x420 pixels). The physical quantities (mass density and momentum) are determined at each node of position $\vec{r}$ by integration in a circular domain of radius $\sigma=4 \mathrm{w}$ using a gaussian kernel

$$
G(\vec{r})=V_{w}^{-1} e^{\frac{-r^{2}}{2 w^{2}}}
$$


where, $V_{w}$ is a constant determined to satisfy $\int G(\vec{r}) d \vec{r}=1$. For a 2D problem we get

$$
\int_{0}^{2 \pi} \int_{0}^{\sigma} V_{w}^{-1} e^{\frac{-r^{2}}{2 w^{2}}} r d r d \theta=1
$$

and,

$$
V_{w}=\int_{0}^{2 \pi} d \theta\left[-w^{2} e^{\frac{-r^{2}}{2 w^{2}}}\right]_{0}^{\sigma}=2 \pi\left[-w^{2} e^{\frac{-r^{2}}{2 w^{2}}}\right]_{0}^{\sigma}=2 \pi w^{2}\left(1-e^{-8}\right)
$$

The mass density field is obtained from:

$$
\rho(\vec{r}, t)=\sum_{i} m_{i} G\left(\vec{r}-\vec{r}_{i}\right)
$$

By denoting $\alpha$ the $x$ or $y$ component, the coarse-grained momentum density vector is

$$
\overrightarrow{p_{\alpha}}(\vec{r}, t)=\sum_{i} m_{i} \vec{v}_{i \alpha} G\left(\vec{r}, \vec{r}_{i}\right)
$$

and the velocity field is

$$
\overrightarrow{V_{\alpha}}(\vec{r}, t)=\frac{\overrightarrow{p_{\alpha}}(\vec{r}, t)}{\rho(\vec{r}, t)}
$$

In this method, the higher $\frac{w}{\bar{d}}$ the more homogeneous the velocity field is. Note also that as this method is a non-local approach the computation effort increases with $\frac{w}{\bar{d}}$ (for fixed value of $\Delta x$ ). We set $w=1.5 \bar{d}$ that is in the range of values proposed by Weinhart et al. [42] and gives velocity fields which compares well with PIV results. 
a)

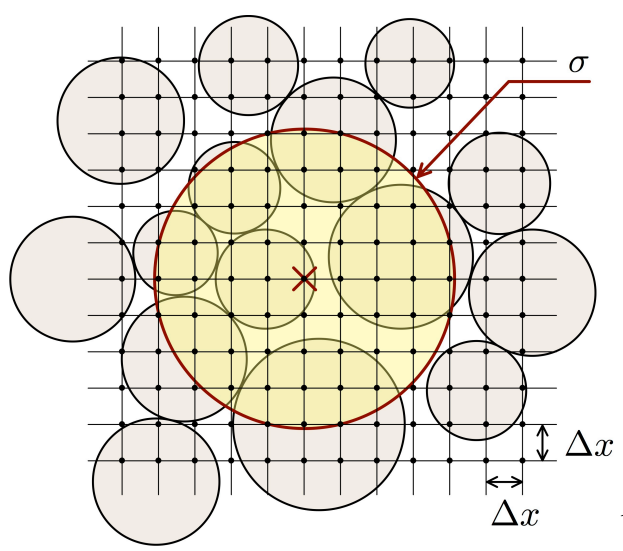

b)

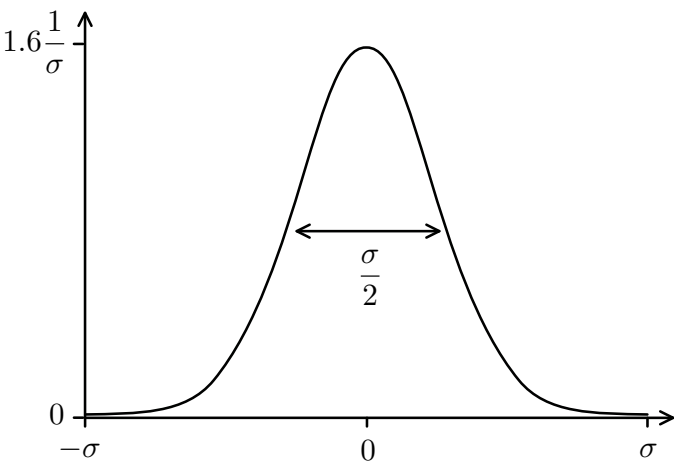

Fig. 5 Coarse-graining method. a) Meshing of the domain; the computation of physical quantities (velocity, stresses, and particle volume fraction) are performed at each node within the characteristic size $\sigma$. b) Illustration of the gaussian kernel (Eq.

4).

\section{Experimental results versus DEM simulations}

In this section we compare experimental and numerical pull-off tests to analyze the force transmission and the localization of the particle mobility. We discuss the origins of the spatiotemporal fluctuations due to the intruder's motion in the granular bed.

\subsection{Drag force}

In both experimental and numerical cases, the force exerted by the granular material on the intruder during its vertical ascension presents a heterogeneous profile due to a succession of fluctuations (Fig. 6). Although the amplitude of these fluctuations are higher for simulations, we clearly see a good agreement between the experimental and numerical general trends. The origin of these fluctuations was studied by [44] for the penetration a cylindrical punch into a dense cohesive granular bed and by Gravish et al. [45] for an intruder moved horizontally. Both 
studies attribute the origin of the fluctuations to the phenomenon of packing dilatancy which is known to strongly depends on the initial packing fraction compared to its critical value in the steady state [46].

The drag force profile (Fig. 6) can be decomposed into two parts. The first part, located in the vicinity of the bottom of the cell (bottom zone) corresponds to the beginning of the pull-off of the intruder. It starts by the development of a high force peak followed by its disappearance, and then a new loading step. The second part is marked by a succession of loading/rupture events whose intensity relaxes as the intruder reaches the free surface. This behavior corresponds to a global relaxation of the drag force. For the velocity of one millimeter per second chosen is this study, the peaks are spaced apart of approximately a grain radius $(\bar{d} / 2)$ corresponding to frequency of $1 \mathrm{~Hz}$. For higher velocities, Mandato et al. [28] shown that this frequency also depends on the velocity of the intruder due to dynamics effects.

The force network (normal component) is shown in Fig. 6 for a height of intruder of $34 \mathrm{~mm}$. We note a strong localization of the largest force chains that starts from upper part of the intruder. The force transmission from the intruder to the granular material follows preferential paths. However, the state of the network experiences loading/rupture events as evidenced by the fluctuations measured on the drag force. Fig. 7 shows an example of a loading/rupture event for the intruder located in the lower part of the cell. A large zone of the packing above the intruder is disturbed leading to a decrease in contact connectivity. The transient rupture observed on the drag force is due to the breakage of the force paths. Interestingly, force chains breakage is localized in the vicinity of the intruder and is not due to a long-range failure. In Fig. 7 we see that the breakage of the main force chains is initiated by the displacement of few lateral grains directly in contact with the intruder. A new preferential path gradually forms again and other contacts percolate from the intruder to the walls inducing a new increase in the drag force. 
While this description of the fluctuations in drag force remains valid during the pull-off test, their magnitude depends on the distance of the intruder to the free surface. It worth noting that in the upper part of the cell (Fig. 6) the amplitude of the force fluctuations decreases almost linearly. In the top layer, these fluctuations vanish with the overall relaxation of the drag force until a depth of nearly $45 \mathrm{~mm}$ from the free surface corresponding to a height of $105 \mathrm{~mm}$. Note that at this position the distance between the end of the intruder and the walls is approximatively the same as the distance between the intruder and the free surface. In this top-layer the magnitude of the force depends linearly with the depth and the force network becomes isotropic. It worth noting that in this zone the force network does not percolate from the intruder to the wall. For a weakly cohesive granular material, the thickness of this layer was already identified as a characteristic length by Mandato et al. [28].
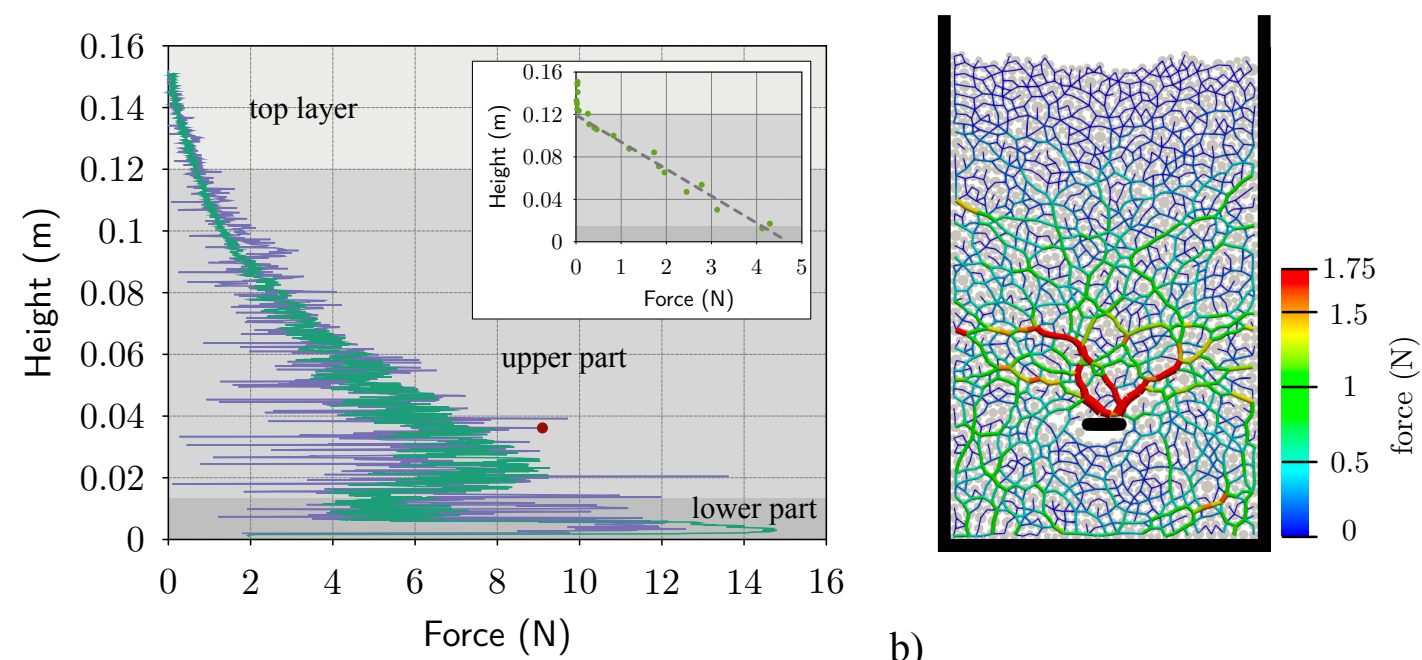

a)

Fig. 6 a) Experimental drag force profile (green solid line) vs DEM (blue solid line).

The inset shows the evolution the amplitude of loading/rupture events. b) Simulation snapshot showing the force network in color levels (the thickness of segments is proportional to the magnitude of the normal force). The red dot on the graph shows the value of the force for the corresponding position of the intruder in b). 
a)

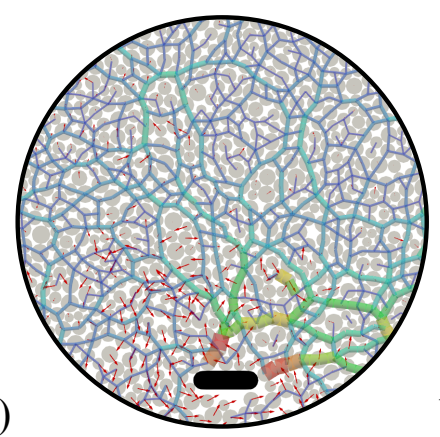

b)

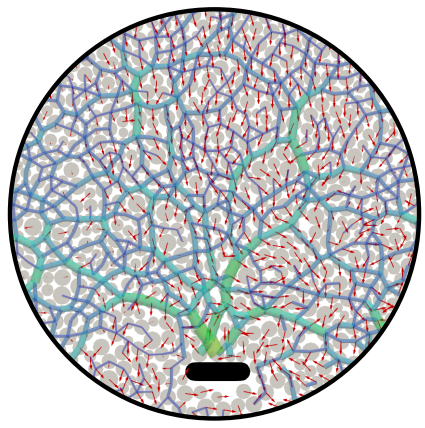

c)

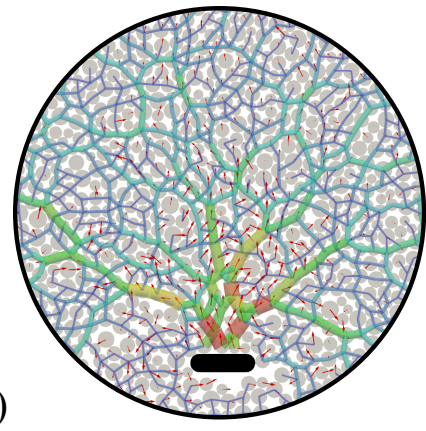

Fig. 7 Three magnifications showing a loading/rupture event in the vicinity of the intruder.

The force network is plotted with the same color gradient as in Fig. 7. The velocity field is shown in red arrows with length proportional to $\bar{d} / V$, where $V$ is the velocity of the intruder.

\section{2. Particle velocity field}

In this section we rely on the coarse-grained DEM velocity fields to investigate the kinematics of particles (see section 2.3 Fig. 5). Fig. 8 shows $v^{*}$ the magnitude of coarse-grained velocities normalized by $V$ for five different intruder positions during loading phases just before the rupture of force chains.

a)

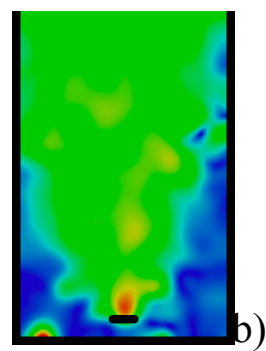

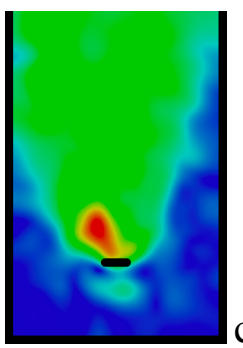

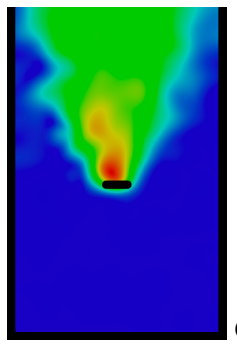

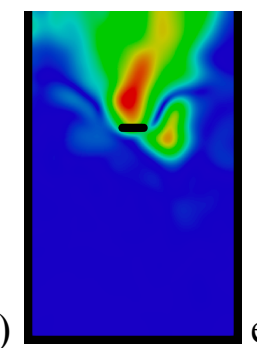

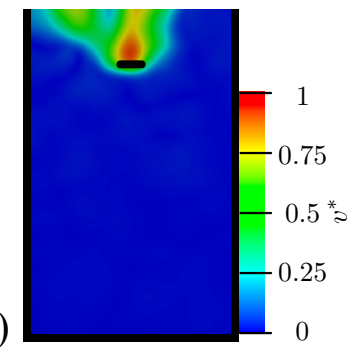

Fig. 8 Visualization of the intruder's influence zone. The velocity magnitude normalized by $V$ (velocity of intruder) is shown in color gradient for 5 different positions: a) in the lower part, b) c) d) in the upper part and e) in the top layer. 
Different modes of particle rearrangement can be identified during the vertical ascension of the intruder. This motion induces an intermittent granular mobility from the position of the intruder to the free surface. Four specific cases can be identified on Fig. 8:

a) The particles in the blue zone $\left(v^{*}<0.25\right)$ are immobile or experienced weak displacements due to local rearrangements.

b) In the red zone above the intruder $\left(v^{*}>0.75\right)$, the dragged particles have a velocity close to that of the intruder $V=1.210^{-3} \mathrm{~m} \mathrm{~s}^{-1}$. During a loading event, this red zone propagates in front of the intruder along the vertical axis. When a breakage occurs, this zone reduces to a cluster of particles directly in contact with the intruder. Finally, as shown in Fig. 8 and 9, the size of the red zone increases with the vertical position of the intruder, except in the top layer when approaching the free surface.

c) During loading phases the grains are displaced in a conic domain above the intruder. This zone corresponds to a range of velocities $0.25<v^{*}<0.75$ displayed in green in Fig. 8. For $V=1.210^{-3} \mathrm{~m} \mathrm{~s}^{-1}$ the opening angle of the cone is almost constant with time and equals to $\beta=60^{\circ}$. Due to the presence of the lateral walls, for intruder height below $H_{\beta}=\frac{1}{2}\left(L_{c}-L\right) \tan \beta$ this cone is truncated (in our case $H_{60}=0.078 \mathrm{~m}$ ). We note in Fig. $8 \mathrm{a}$ and $8 \mathrm{~b}$ that in the green zone all particles are displaced, including those in contact with the walls. When the intersection of the cone with the walls reaches the free surface (Fig. 8c), the particle mobility does not percolate up to the walls.

d) Ruptures in the force network trigger convective patterns in the vicinity of the ends of the intruder (Fig. 10). The particles laterally bypass the intruder before avalanching underneath in an inverse-heap shaped hole. The flow of particles is intermittent and occurs on both sides with respect to the vertical axis. The occurrence of the discharge of particles may be in or out of phase leading to fluctuations in the hole size under the intruder. As an illustration we chose a situation where the avalanches on both sides of 
intruder occurs in phase (Fig. 10a) and out of phase (Fig. 10b). Fig. 10a and Fig. 10b also show velocity vortices for PIV and coarse-graining, respectively. These mobility zones, based on the velocity fields analysis, are summarized on the scheme Fig. 10c. The observed vortices are comparable to those already evidenced in papers dealing with the so-called granulence phenomenon [47][48][49] which is an analogue of the turbulence for granular flows. Note that, these vortices are intermittent and do not last sufficient time to generate a persistent rotational flow of particles. Contrary to the vortices observed in the papers of Radjai et al. [47], Miller et al.[48], Combe et al.[49] which appear randomly in homogeneous shear flows, in our case the intruder localizes these labile structures in the vicinity of the tips.

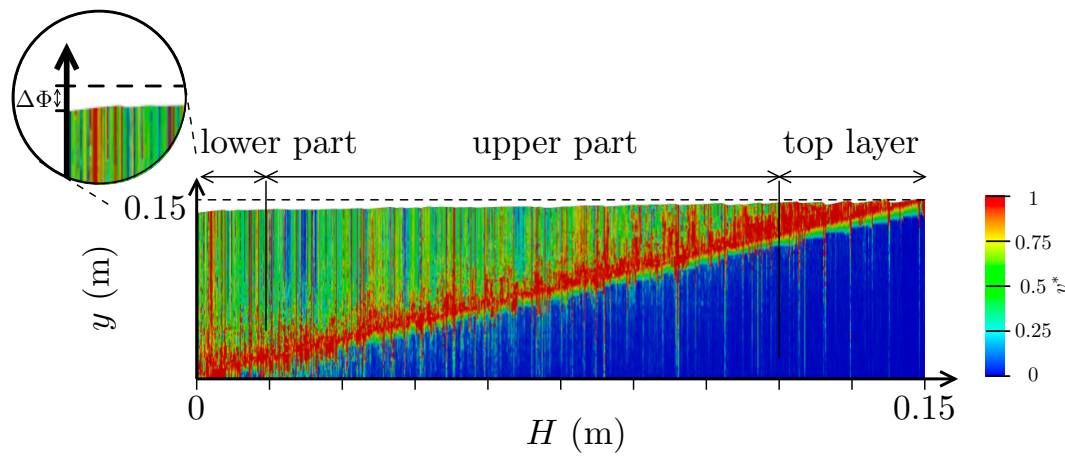

Fig. 9 Normalized vertical velocity along a vertical line $(y)$ in the center of the cell as a function of the position $H$ of the intruder. Top-left corner, magnification of the onset showing the dilatancy $\Delta \Phi$ of packing. 
a)

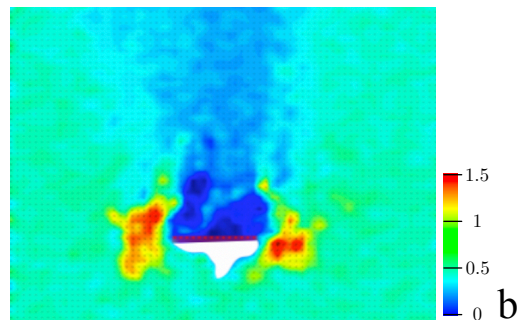

b)

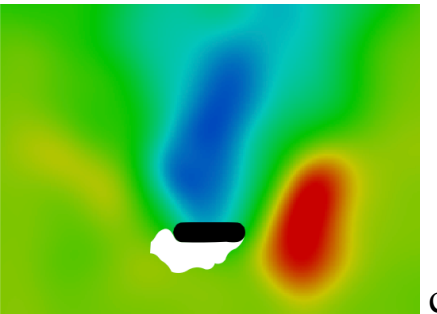

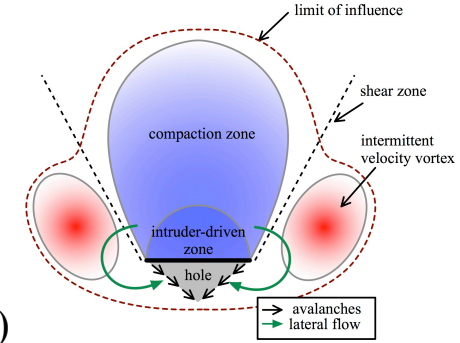

Fig. 10 Magnification of the velocity field normalized by $V$ determined in the local frame of the intruder during its vertical ascension using a) PIV methods and b) coarse graining (plotted with the same color gradient). c) Scheme of the pattern of mobility around the intruder.

\section{Discussion}

In order to clarify the ability of the intruder to transmit mobility to particles through the contact network, Fig. 11 shows the normal force network superimposed on the coarse-grained velocity field (with color ranged in $\log$ scale). This representation enlightens the spatio-temporal correlations between force transmission and particle mobility. Indeed, during its vertical ascension, the intruder supplies energy to the granular medium that is stored in the elastic interactions at contact scale and is dissipated through the friction, damping and rearrangement of particles. 
a)
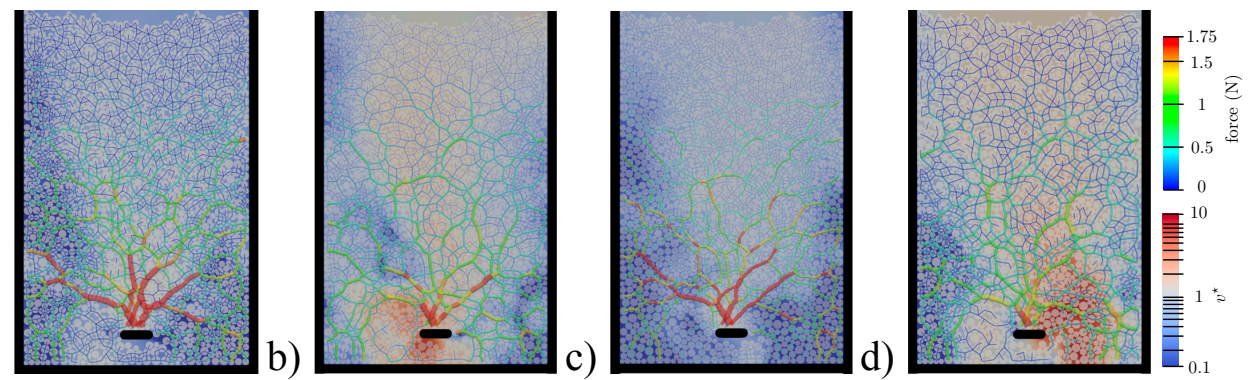

Fig. 11 Four snapshots showing two successive loading/rupture events. The force network is superimposed on the coarse grained velocity field. The velocity scale is in logarithmic scale.

During the loading phases, the normal force network polarizes along preferential force chains (Fig. 11a and Fig. 11c). These chains are limited by the walls, creating an upward front at the vertical of the intruder but also are at the origin of transient arches joining the intruder-driven particles (Fig. 11a, 11c) to the lateral particles in contact with the walls. The velocity field is greater in the zones where the forces are weak except for particles localized in front of the intruder (Fig. 12a). The ratio between the tangential force and its maximum value is denoted $\mu^{*}=F_{t} / F_{t}^{\text {slip }}$ which is a measure of the friction mobilization within the Coulomb range [50] (note that in this paper the particle-particle and particle-walls friction coefficients are the same). With this definition $\mu^{*}$ equals 1 for particle at the slip threshold (Fig. 12b and Fig. 12c). In the sample, the displacement occurs even if the contact's slip threshold is not reach (Fig. 12b). Interestingly, this is true not only for particles in the bulk but also for those in contact with the walls. As an illustration, Fig. 12c shows corresponding values of $\mu^{*}$ for all particles together with the Coulomb yield surface (dotted lines). It worth noting the slip threshold is reached for less than $20 \%$ of the contacts.

During this transient phase of loading, some particles focuses the forces on the intruder (Fig. $7 \mathrm{~b}$ and $7 \mathrm{c}$ ) playing the role of keystones in-between lateral arches that percolates to the walls. An increment of intruder displacement increases the magnitude of the forces involved in these arches. Concomitantly, this motion of the intruder leads to incremental particle displacements. 
The strain localized in a shear bands (Fig. 10c) and the particle motion only implies the conic green zone (Fig. 8). This leads to an increase of the volume of the hole below the intruder as the granular material underneath is immobile.

a)

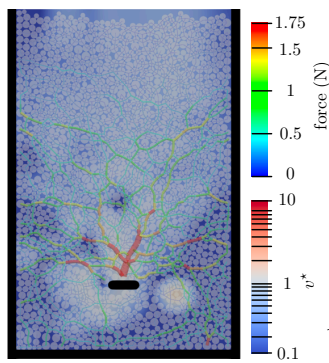

b)

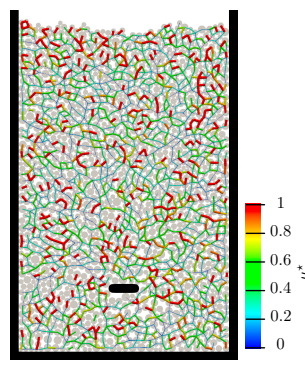

c)

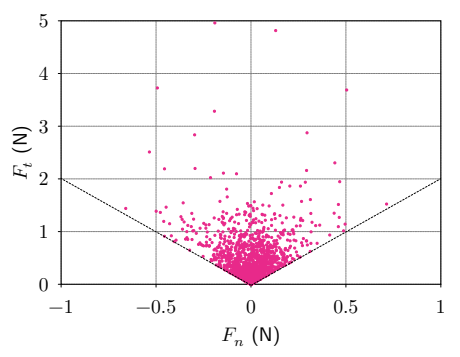

Fig. 12 a) Normal force network superimposed with the velocity field. b)

Representation of $\mu^{*}$ for all contacts at same time as in a). c) Corresponding values of normal vs. tangential forces at each contact (cone of Coulomb).

The rupture occurs when a particle in contact with an end of the intruder drop underneath. This particle is ejected from its keystone position leading to a sudden displacement on a length greater than a diameter which disconnects the force chains. This phenomenon can be viewed as a disjunction in the force network that occurs at large scale. Consequently, avalanches appear in the free volume under the intruder on one or both sides together with a relaxation at long range of particles above the intruder. The contact network reforms again due to rearrangements of particles, conducting to a new static state under the intruder and a strengthening of the force network. As already evidenced on the fluctuations of the drag force (Sec. 3.1) this sequence of loading/rupture events synchronized with intermittent flows (Sec. 3.2). This phenomenon repeats along the rise of the intruder with a magnitude that depends on the position of the vertical intruder. 
In the lower part (Fig 6a), the opening of hole under the intruder leads to a local densification of the particle bed which resists to the motion of the intruder and induce a first loading/rupture event. This resistance is at the origin of force chains of high magnitudes. The rupture of these chains is a critical event at the onset of the largest observed displacements of the whole test. The observed decrease in particle solid fraction can be attributed to a global dilatancy (Fig. 9) $[44,45,51]$. Fig. 13 shows $\bar{\mu}^{*}$ the average value of $\mu^{*}$ for all contacts in the bulk (pink dots) or at the walls (green dots), as a function of the position of the intruder. We note that $\bar{\mu}^{*}$ is well below 1 (sliding value) and is maximum in the lower part.

In the upper part of the cell, as long as the position of the intruder is lower than $H_{\beta}$, particles rearrange from the intruder to the walls. In this zone, the force network percolates to the walls during the loading phases. In the bulk and at the walls $\bar{\mu}^{*}$ fluctuates around constant values lower than 1 indicating that motion of particles does not occurs at the yield friction value for all contacts. This shows that the strain occurs in-between clusters of particles [52]. In Fig 12b we note also that the largest values of $\mu^{*}$ occurs for the particles in motion whereas the lowest values can be associated with particles that contributes to strong force chains.

For positions of the intruder greater than $H_{\beta}$, the force network still percolates but the mobility of particles is lost in the vicinity of the walls. Above $H_{\beta}$, both values of $\bar{\mu}^{*}$ for the bulk and the walls decrease toward $\bar{\mu}^{*} \approx 0.24$ (Fig. 13). Finally, when the intruder reaches the top-zone the connexity of the force chains is lost and the magnitude of forces becomes lower compared to the average normal force in the bed below the intruder. 


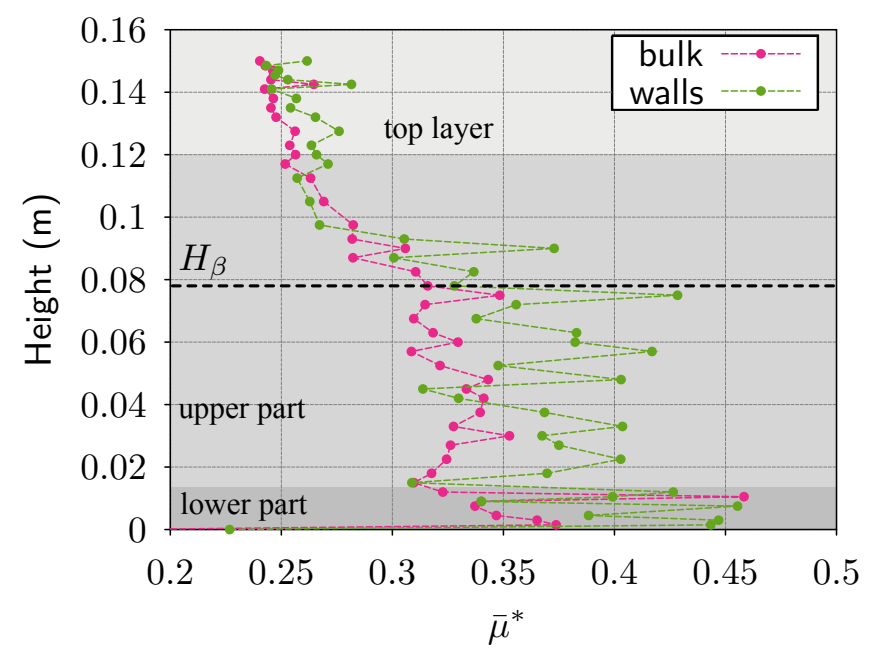

Fig. 13 Average friction $\bar{\mu}^{*}$ of $\mu^{*}$ in the bulk and at the walls as a function of the position of the intruder.

\section{Conclusion}

In this paper, we present a methodology for the study of particle mobility and force transmission induced by an intruder immersed in a dense granular packing. This methodology relies on experimental and DEM numerical simulations to better understand the evolution of the drag force acting on an intruder and its consequences on particle's rearrangements.

We found a good agreement between experimental and numerical evolution of the drag force. Although the simulations are in 2D the PIV fields compared well with coarse grained fields derived from the position and velocities computed at a particle scale. One major advantage of the DEM has been to provide detail analysis at the contact scale in terms of force transmission and mobilization of friction; both in the bulk and in the vicinity of the walls.

During a pull-off test of the intruder, loading/breaking events were evidenced. At large scale, the rupture of the force network appears to be a consequence of the ejection of particles directly 
in contact with the ends of the intruder. However, the dilatancy observed at global scale does not seems to be at the origin of this phenomenon.

The velocity field and the force network are influenced by the presence of the walls. This influence affects the magnitude of forces and velocities but also has an impact on their spatial extension. Two characteristic lengths were evidenced above which the velocity field and the force network decorrelates from the influence of the walls. The insights provided by the numerical simulations call also for new investigations on spatio-temporal correlations in such a system.

These two characteristic lengths allow us to distinguish several zones which can be associated with specific granular behaviors typologies. The detailed characterization of these zones is an important sizing aid for the design of processes. For example, in the hydrostatic zone the particle inertia is high and the force network is nearly isotropic with a low friction mobilization limiting abrasion phenomena. There is a potential benefit to maximize this area in mixing operation. On the contrary, for kneading and wet agglomeration in which it is necessary to control the growth of particles, it is more suitable to promote high friction mobilization. Finally, for higher input energy as in attrition mill the zone below hydrostatic zone should also be privileged.

Ongoing works aims to provide a detailed parametric study of the influence of material parameters such as friction, stiffness or cohesion. The finite size (diameter of particles compared to the cell size) and dynamics (intruder velocities, cyclic loading) effects will also be investigated with this approach. Finally, one major concern will be to vary the dimension and shape of the intruder to provide design assistance and help to process control.

\section{Aknowledgments}


The authors thank the project DEFI Blé Dur, the MetaProgram GloFoodS INRAE/CIRAD fand the INRAE project ANS PowFlow for their financial supports. The results presented in this paper are part of DEFI Blé dur project (2016-2022) that has received funding from Programme d'investissement d'avenir (PIA).

The numerical simulations were performed with the support of MESO@LR-Platform at the University of Montpellier.

\section{Compliance with ethical standards}

Conflict of interest The authors declare that they have no conflict of interest.

\section{References}

1. Radl, S., Brandl, D., Heimburg, H., Glasser, B.J., Khinast, J.G.: Flow and mixing of granular material over a single blade. Powder Technology, 226, 199-212, (2012)

2. Chandratilleke, G.R., Yu, A.B., Bridgwater, J.: A DEM study of the mixing of particles induced by a flat blade. Chemical Engineering Science, 79, 54-74 (2012)

3. Moreno Juez, J., Artoni, R., Cazacliu, B.: Monitoring of concrete mixing evolution using image analysis. Powder Technology, 305, 477-487 (2017)

4. Muzzio, F.J., Robinson, P., Wightman, C., Brone, D.: Sampling practices in powder blending. International Journal of Pharmaceutics, 155(2), 153-178 (1997) 
5. Barling, D., Morton, D.A.V., Hapgood, K.: Pharmaceutical dry powder blending and scaleup: Maintaining equivalent mixing conditions using a coloured tracer powder. Powder Technology, 270, B, 461-469 (2015)

6. Cuq, B., Berthiaux, H., Gatumel, C.: Powder mixing in the production of food powders. In Handbook of Food Powders, Processes and Properties, Woodhead Publishing Series in Food Science, Technology and Nutrition, 200-229 (2013)

7. Shenoy, P., Xanthakis, E., Innings, F., Jonsson, C., Fitzpatrick, J., Ahrné, L.: Dry mixing of food powders: Effect of water content and composition on mixture quality of binary mixtures, Journal of Food Engineering, 149, 229-236 (2015)

8. Kolb, E., Legué, V., Bogeat-Triboulot, M.B.: Physical root-soil interactions. Physical Biology, 14(6), 065004 (2017)

9. Fakih, M., Delenne, J. Y., Radjai, F., Fourcaud, T.: Root growth and force chains in a granular soil. Physical Review E, 99(4), 042903 (2019)

10. Ammarcha, C., Gatumel, C., Dirion, J.L., Cabassud, M., Mizonov, V., Berthiaux, H.: Powder flow and mixing in a continuous mixer operating in either transitory or steady-state regimes: Mesoscopic Markov chain models. Powder Technology, 346, 116-136 (2019)

11. Remy, B., Khinast, J.G., Glasser, B.J.: Wet granular flows in a bladed mixer: Experiments and simulations of monodisperse spheres. Aiche Journal, 58(11), 3354-3369 (2012) 
12. Mandato, S., Ruiz, T., Cuq, B.: What is Janssen's length doing in an agglomerator? Powder Technology, 238, 56-63 (2013)

13. Daraio, D., Villoria, J., Ingram, A., Alexiadis, A., Stitt, E.H., Marigo, M.: Validation of a discrete element method (DEM) model of the grinding media dynamics within an attritor mill using positron emission particle tracking (PEPT) measurements. Applied SciencesBasel, 9(22), $4816(2019)$

14. Motte, J.C., Delenne, J.-Y., Rouau, X., Mayer-Laigle, C.: Mineral-vegetal co-milling: An effective process to improve lignocellulosic biomass fine milling and to increase interweaving between mixed particles. Bioresource Technology, 192, 703-710 (2015)

15. Yaraghi, A., Ebrahimi, M., Ein-Mozaffari, F., Lohi, A.: Mixing assessment of noncohesive particles in a paddle mixer through experiments and discrete element method (DEM). Advanced Powder Technology, 29(11), 2693-2706 (2018)

16. Ebrahimi, M., Yaraghi, A., Ein-Mozaffari, F., Lohi, A.: The effect of impeller configurations on particle mixing in an agitated paddle mixer. Powder Technology, 332, $158-170(2018)$

17. Pantaleev, S., Yordanova, S., Janda, A., Marigo, M., Ooi, J.Y.: An experimentally validated DEM study of powder mixing in a paddle blade mixer. Powder Technology, 311, 287-302 (2017) 
18. Cuq, B., Mandato, S., Jeantet, R., Saleh, K., Ruiz, T.: Agglomeration/granulation in food powder production. In B. Bhandari, N. Bansal, M. Zhang, and P. Schuck, (Eds.), Handbook of Food Powders. Woodhead Publishing Series in Food Science, Technology and Nutrition, 255, pp. 150-177 (2013)

19. Rondet, E., Delalonde, M., Ruiz, T., Desfours, J.P.: Fractal formation description of agglomeration in low shear mixer, Chemical Engineering Journal, 164, 376-382 (2010)

20. Kolb, E., Mazozi, T., Clément, E., Duran, J.: Force fluctuations in a vertically pushed granular column. The European Physical Journal B, 8(3), 483-491 (1999)

21. Deboeuf, S., Dauchot, O., Staron, L., Mangeney, A., Vilotte, J.-P.: Memory of the unjamming transition during cyclic tiltings of a granular pile, Physical Review E, 72, $051305(2005)$

22. Costantino, D. J., Scheidemantel, T. J., Stone, M. B., Conger, C., Klein, K., Lohr, M., Schiffer, P.: Starting to move through a granular medium. Physical review letters, 101(10), $108001(2008)$

23. Seguin, A., Bertho, Y., Martinez, F., Crassous, J., Gondret, P.: Experimental velocity fields and forces for a cylinder penetrating into a granular medium. Physical Review E, 87(1), $012201(2013)$ 
24. Seguin, A., Coulais, C., Martinez, F., Bertho, Y., Gondret, P.: Local rheological measurements in the granular flow around an intruder. Physical Review E, 93, 012904 (2016)

25. Fullard, L.A., Breard, E.C.P., Davies, C.E., Godfrey, A.J.R., Fukuoka, M., Wade, A., Dufek, J., Lube, G.: The dynamics of granular flow from a silo with two symmetric openings. Proc. R. Soc. A. 475, 20180462 (2019)

26. Andreotti, B., Forterre, Y., Pouliquen, 0.: Granular media: between fluid and solid, Cambridge University Press (2013)

27. Athani, S., Rognon, P.: Inertial drag in granular media. Physical Review Fluids, 4(12), $124302(2019)$

28. Mandato, S., Cuq, B., Ruiz, T.: Experimental study of vertical stress profiles of a confined granular bed under static and dynamic conditions. The European Physical Journal E, 35(7), $56(2012)$

29. Ovarlez, G., Fond, C., \& Clément, E.: Overshoot effect in the Janssen granular column: a crucial test for granular mechanics. Physical Review E, 67(6), 060302 (2003)

30. Blair, D.L., Mueggenburg, N.W., Marshall, A.H., Jaeger, H.M., Nagel, S.R.: Force distributions in three-dimensional granular assemblies: Effects of packing order and interparticle friction. Physical review E, 63(4), 041304 (2001) 
31. Sarno, L., Carravetta, A., Tai, Y.C., Martino, R., Papa, M.N., Kuo, C.Y.: Measuring the velocity fields of granular flows. Employment of a multi-pass two-dimensional particle image velocimetry (2D-PIV) approach. Advanced Powder Technology, 29(12), 3107-3123 (2018)

32. Radjaï, F., Dubois, F.: Discrete-element modeling of granular materials. Wiley-ISTE, New-York (2011)

33. Allen, M.P., Tildesley, D.J.: Computer simulation of liquids. Oxford university press (2017)

34. Rognon, P.G., Roux, J.N., Naaim, M., Chevoir, F.: Dense flows of cohesive granular materials. Journal of Fluid Mechanics, 596, 21-47 (2008)

35. Gilabert, F. A., Roux, J. N., Castellanos, A.: Computer simulation of model cohesive powders: influence of assembling procedure and contact laws on low consolidation states. Physical review E, 75(1), 011303 (2007)

36. Cundall, P.A., Strack, O.D.: A discrete numerical model for granular assemblies. Geotechnique, 29(1), 47-65 (1979)

37. Mello, N.M., Paiva, H.A., Combe, G., Atman, A.P.F.: Fingering phenomena during graingrain displacement. Computational Particle Mechanics, 4(2), 153-164 (2017) 
38. Campbell, C.S.: Granular shear flows at the elastic limit. Journal of fluid mechanics, 465 , 261-291 (2002)

39. Voivret, C., Radjai, F., Delenne, J.-Y., El Youssoufi, M.S.: Multiscale force networks in highly polydisperse granular media. Physical review letters, 102(17), 178001 (2009)

40. Marinack, M.C., Musgrave, R.E., Higgs, C.F.: Experimental Investigations on the Coefficient of Restitution of Single Particles. Tribology Transactions, 56, 572-580 (2013)

41. Delaplace, G., Thakur, R.K., Bouvier, L., André, C., Torrez, C.: Dimensional analysis for planetary mixer: Mixing time and Reynolds numbers. Chemical Engineering Science, 62(5), 1442-1447 (2007)

42. Weinhart, T., Labra, C., Luding, S., Ooi, J. Y.: Influence of coarse-graining parameters on the analysis of DEM simulations of silo flow. Powder technology, 293, 138-148 (2016)

43. Algarra, N., Karagiannopoulos, P. G., Lazarus, A., Vandembroucq, D., Kolb, E.: Bending transition in the penetration of a flexible intruder in a two-dimensional dense granular medium. Physical Review E, 97(2), 022901 (2018)

44. Miyai, S., Kobayakawa, M., Tsuji, T., Tanaka, T.: Influence of particle size on vertical plate penetration into dense cohesionless granular materials (large-scale DEM simulation using real particle size). Granular Matter, 21(4), 105 (2019) 
45. Gravish, N., Umbanhowar, P. B., Goldman, D. I: Force and flow transition in plowed granular media. Physical review letters, 105(12), 128301 (2010)

46. Guyon, E., Delenne, J.-Y., Radjai, F., Butler, E.: Built on Sand-the Science of Granular Materials. Ed. Mit Press, Boston (2020).

47. Radjaï, F., \& Roux, S.: Turbulent-like fluctuations in quasistatic flow of granular media. Physical Review Letters, 89(6), 064302 (2002)

48. Miller, T., Rognon, P., Metzger, B., \& Einav, I.: Eddy viscosity in dense granular flows. Physical Review Letters, 111(5), 058002 (2013)

49. Combe, G., Richefeu, V., Stasiak, M., \& Atman, A. P.: Experimental validation of a nonextensive scaling law in confined granular media. Physical Review Letters, 115(23), $238301(2015)$

50. Radjaï, F., Jean, M., Moreau, J., Roux, S.: Force distributions in dense two-dimensional granular systems. Physical Review Letters, 77, 274-277 (1996)

51. Seguin, A.: Experimental study of some properties of the strong and weak force networks in a jammed granular medium. Granular Matter, 22, 48 (2020)

52. Houdoux, D., Nguyen, T.B., Amon, A., Crassous, J.: Plastic flow and localization in an amorphous material: Experimental interpretation of the fluidity. Physical Review E, 98, $022905(2018)$ 
